\title{
50. SPLITTING AND SUDDEN OUTBURSTS OF COMETS AS INDICATORS OF NONGRAVITATIONAL EFFECTS
}

\author{
E. M. PITTICH \\ Astronomical Institute, Slovak Academy of Sciences, Bratislava, Czechoslovakia
}

\begin{abstract}
It is shown that sudden outbursts of comets, or even complete splitting of their nuclei into several parts, are rather frequent phenomena that need to be accounted for in the evolution of cometary orbits. The distribution of the points in which such events occur indicates that the tidal action of the Sun and Jupiter, and also solar radiation, are much more probable causes of these effects than are collisions with the asteroids. Splitting is found to be relatively more frequent for comets moving in hyperbolic orbits. The observed velocities of separation indicate that the disruption of the nucleus provides an effective mechanism for the ejection of the fragments from longperiod into hyperbolic orbits, augmenting the loss by planetary perturbations. The outbursts can be responsible for smaller changes in the orbits, which after repetition might produce a quasi-secular variation.
\end{abstract}

\section{Anomalous Changes in Comets}

Anomalous changes - sudden outbursts of comets and complete splitting of their nuclei into several parts - are rather frequent phenomena in cometary evolution. Stefanik (1966) gives 13 well-documented cases of splitting of nuclei, and Vsekhsvyatskij (1966) gives more than 50 cases of observed sudden changes in the brightness of comets. During the last two centuries at least 40 comets appear to have suddenly increased in brightness shortly before their discovery, as was shown by Pittich (1969) on the basis of an analysis of the distribution of time intervals in which the comets remained undiscovered in spite of favourable observing conditions. The results have proven the reality of sudden changes in cometary brightness, even at considerable distances from the Sun. Only a part of the observed abrupt changes in brightness can be attributed to systematic errors in the estimates of the magnitudes.

These anomalies related to the evolution of comets prove that there exist external factors affecting comets. The following factors might be considered: the tidal action of the Sun and planets, especially Jupiter; collisions of comets with asteroids; and solar radiation. Their effect is spatially restricted, and anomalies in comets depend on the degree of intensity of these factors. The complete splitting of a comet nucleus can be considered as the extreme event.

\section{Space Distribution of the Anomalies}

It was Harwit (1968) who first considered the spatial distribution of the points in which the splitting of cometary nuclei occurred. The author (Pittich, 1971) gives similar data for nine further cases and for a number of cometary outbursts; the two figures presented here are taken from this paper. The space distribution of the splitting of 19 cometary nuclei is plotted in Figure $1 ; r$ is the distance from the Sun, $d$ the distance from the ecliptic plane (full line), in astronomical units. Positive values of $d$ 


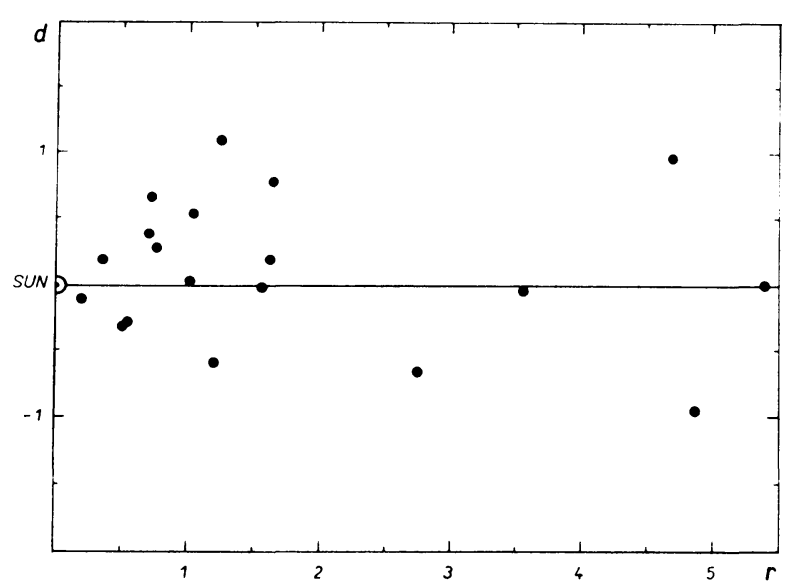

Fig. 1. Positions of comets at the time of splitting.

indicate positions north of the ecliptic, negative values south of it. Figure 2, arranged in the same manner as Figure 1, shows the positions of the outbursts, which occurred shortly before (open circles) and after comet discovery (closed circles).

If selection effects of comet discovery are taken into account, the distribution of the points in Figures 1 and 2 indicates that the tidal action of the Sun and Jupiter, and

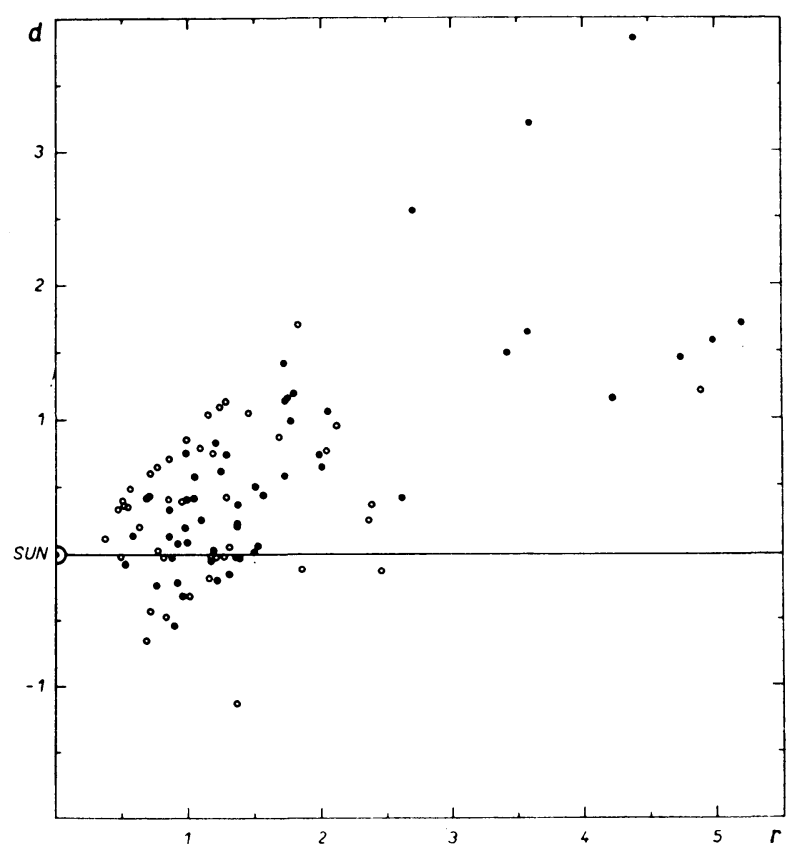

Fig. 2. Positions of comets at the time of outbursts. Open circles: hypothetical outbursts preceding discovery. Closed circles: observed outbursts after discovery. 
solar radiation, are much more probable causes of cometary splitting and outbursts than collisions with the asteroids. Explanation of the splitting and outbursts as due to collisions with asteroidal particles would require a concentration of the points within the main asteroid belt, i.e., roughly between $r=2.2$ and $r=3.2 \mathrm{AU}$. Although this region is beyond the limits of observability of many comets, there appears to be a gap of avoidance rather than any concentration. On the contrary, a secondary maximum located outside the belt, nearer to the distance of Jupiter from the Sun, is indicated.

\section{Indicators of Nongravitational Effects}

Splitting is found to be relatively more frequent for comets moving in hyperbolic orbits than for the others. Approximately $6 \%$ of the known cometary orbits are hyperbolic, while among 19 comets with observed splitting of the nucleus, eight (i.e., $42 \%$ ) belong to this category. This fact can be explained by the assumption that the orbit of the observed part of the nucleus has been affected by splitting.

This assumption is supported by the observed velocities of separation. They range from 2 to $40 \mathrm{~m} \mathrm{~s}^{-1}$, with a characteristic value of about $15 \mathrm{~m} \mathrm{~s}^{-1}$ (Sekanina, 1966; Stefanik, 1966). These observed velocities lie within the range of the theoretical heliocentric velocity increments (Table I) necessary for the corresponding orbital changes of 'new' comets in Oort's sense ( $a \sim 1000 \mathrm{AU})$. The velocity excess between

TABLE I

Velocity increments

\begin{tabular}{lcccc}
\hline$r(\mathrm{AU})$ & $V_{\mathrm{par}}-V_{\mathrm{ell}}\left(\mathrm{m} \mathrm{s}^{-1}\right)$ & & $\begin{array}{l}V_{\mathrm{hyp}}-V_{\mathrm{par}} \\
\left(\mathrm{m} \mathrm{s}^{-1}\right)\end{array}$ \\
\hline & $a=1000$ & $a=100$ & $a=10$ & $a=-1000$ \\
\hline 0.2 & 5 & 47 & 472 & 5 \\
1.0 & 11 & 105 & 1066 & 11 \\
2.0 & 15 & 149 & 1527 & 15 \\
3.0 & 18 & 183 & 1898 & 18 \\
4.0 & 21 & 212 & 2222 & 21 \\
5.0 & 24 & 237 & 2522 & 24 \\
\hline
\end{tabular}

parabolic and hyperbolic orbits, calculated for $a=-1000-$ i.e., for the extreme hyperbolic value observed (Porter, 1961; Marsden, 1966) - also agrees with the observed disruption velocities. As hyperbolic orbits of higher eccentricity have not been observed, it seems that hyperbolic orbits and the disruption of nuclei may have a close connection. The splitting of nuclei provides an effective mechanism, besides planetary perturbations, for the ejection of fragments from long-period into hyperbolic orbits. On the other hand, the splitting of a nucleus indicates the presence of nongravitational forces acting on the motions of comets.

For the compilation of Table I, using the formula

$$
V^{2}=k^{2}\left(\frac{2}{r}-\frac{1}{a}\right)
$$


five values of the semimajor axis have been selected: three for elliptical orbits, one for parabolic, and one for hyperbolic. For elongated elliptical orbits the velocity increment required for ejecting from the solar system comets moving near the Sun is roughly proportional to $a^{-1}$, irrespective of the heliocentric distance $r$ at which the acceleration occurs. On the other hand, the velocity increment necessary for such ejection varies with $r^{1 / 2}$. Therefore, the greatest changes in semimajor axis take place when the comet splits near the Sun. Table I shows that only 'new' comets can suffer this type of nongravitational ejection from the solar system, as the necessary velocity increments are too large if $a$ is of the order of 10 to $100 \mathrm{AU}$.

The splitting of a nucleus, however, is the extreme case of anomalous changes in comets. Greater or smaller outbursts, observed as flares in brightness, occur more frequently. In such events sufficiently strong nongravitational changes in the orbits can hardly be expected. In general, the outbursts can be held responsible only for smaller orbital changes, which after repetition might produce a quasi-secular variation.

The outbursts are about three times more frequent in comets in which an outburst has already been observed previously. This conclusion was obtained from 181 individual comets observed in 1925-1965. At least one outburst was recorded in 28 comets, and more than one outburst in 12 comets (Vsekhsvyatskij, 1966); i.e., the probability of the occurrence of an outburst is $p=0.16$ in the former case and $p=0.43$ in the latter case. Among 19 comets in which splitting of the nucleus occurred, there are nine cases in which outbursts have also been observed (Pittich, 1971), i.e., $p=0.47$. This value is similar to the preceding one. Although the data on observed outbursts must be used with caution, in view of considerable errors in the magnitude data, which can simulate sudden changes in brightness, the statistical difference appears rather significant. A category of comets exists in which anomalous changes tend to repeat during their evolution.

It appears that the splitting and outbursts are very likely to be accompanied by nongravitational changes in cometary orbits. The occurrence of these anomalous phenomena can be used as suitable criteria for the selection of objects in studies of the nongravitational effects on cometary motion.

\section{References}

Harwit, M.: 1968, Astrophys. J. 151, 789.

Marsden, B. G.: 1966, Mem. Br. Astron. Assoc. 40, No. 2.

Pittich, E. M.: 1969, Bull. Astron. Inst. Czech. 20, 251.

Pittich, E. M.: 1971, Bull. Astron. Inst. Czech. 22, 143.

Porter, J. G.: 1961, Mem. Br. Astron. Assoc. 39, No. 3.

Sekanina, Z.: 1966, Bull. Astron. Inst. Czech. 17, 207.

Stefanik, R. P.: 1966, Mem. Soc. Roy. Sci. Liège Ser. 5 12, 29.

Vsekhsvyatskij, S. K.: 1966, Problemy Kometnoj Fotometrii Inf. Byull. No. 10, 3. 